\title{
FinTech y BigTech: Estado de situación sobre las barreras a la entrada y a la innovación en América Latina
}

Revista Latinoamericana de Economía y Sociedad Digital

Issue 1 , agosto 2020

Autores: Esteban Greco, Fernanda Viecens

DOI: $10.53857 /$ THMN7438

Publicado: 10 agosto, 2020

Cita sugerida: Greco, Esteban \& Viecens, Fernanda (2020) "FinTech y BigTech: Estado de situación sobre las barreras a la entrada y a la innovación en América Latina" en Revista Latinoamericana de Economía y Sociedad Digital, Issue 1

Licencia: Creative Commons Atribución-NoComercial 4.0 Internacional (CC BY-NC 4.0)

Tipo: Ensayo

Palabras clave: Defensa de la Competencia, Economía de la innovación, FinTech, Inclusión Financiera, Mercados de medios de pagos

\section{Resumen}

El sector financiero a nivel global se encuentra inmerso en un proceso de transformación, motivado por la incorporación de tecnologías digitales e innovaciones disruptivas que se manifiestan en nuevos productos, jugadores y modelos de negocio. Este escenario ha obligado a los bancos -actores tradicionales- a replantear sus estrategias competitivas y a las autoridades regulatorias a revisar el enfoque con el que fueron concebidas las normas preexistentes. El objetivo de este artículo es identificar barreras que pudieran estar actuando como límites al desarrollo de la innovación en el sector financiero en América Latina y a la entrada de nuevos actores con potencial para generar inclusión financiera. Se documentan y analizan medidas e intervenciones que han llevado a cabo diversas agencias de competencia de la región en años recientes, en muchos casos motivadas por las demandas que impone la innovación tecnológica, por denuncias de jugadores entrantes o por investigaciones abiertas de oficio. 


\section{Introducción}

El sector financiero a nivel global se encuentra inmerso en un proceso de transformación, motivado por la incorporación de tecnologías digitales e innovaciones disruptivas que se manifiestan en nuevos productos, nuevos jugadores y nuevos modelos de negocio. Este escenario ha obligado a los bancos -los actores tradicionales- a replantear sus estrategias competitivas y a las autoridades regulatorias, a revisar el enfoque con el que fueron concebidas las normas preexistentes. Además, los bajos niveles de bancarización de los países emergentes han impulsado el nacimiento de nuevos actores con potencial para generar inclusión financiera y una mayor aceptación de medios de pagos alternativos al dinero físico.

En este contexto, la pandemia de COVID-19 y la necesidad de aislamiento social han puesto en evidencia más que nunca la importancia de contar con una sociedad bancarizada y con acceso a medios de pagos digitales, que ofrecen una reducción de los costos de transacción. Así, la posibilidad de hacer compras de manera sencilla y remota, y acceder a servicios online es más valorada ${ }^{[1]}$, mientras que el pago con código QR o contactless en las transacciones físicas remanentes reduce el riesgo de contagio ${ }^{[2]}$. Consecuentemente, cierta evidencia muestra un aumento importante en el uso de medios de pagos sin transacciones físicas y algunos de los nuevos jugadores reportan un crecimiento sin precedentes ${ }^{[3]}$.

Por el lado de la oferta, la situación trajo desafíos y oportunidades para empresas y personas que pudieran ofrecer y vender servicios online. Nuevos productos y servicios surgieron como resultado de un despliegue de estrategias creativas generado por la necesidad de que las familias se mantuvieran en sus hogares ${ }^{[4]}$.

Sin embargo, este escenario de nuevas oportunidades ha estado restringido a personas y empresas que se encontraban digitalizadas, bancarizadas y con acceso a plataformas de pago digitales. De hecho, una de las debilidades con las que debieron enfrentarse algunos Gobiernos de América Latina frente a la pandemia fue precisamente la alta tasa de uso de dinero físico para realizar transacciones ${ }^{[5]}$. Al mismo tiempo, se evidencia una gran preocupación por una cantidad de empresas y de pequeños productores locales que están sufriendo los efectos de la falta de actividad, algunos de los cuales, de haber estado inmersos en la digitalización, tal vez podrían haber sobrellevado mejor el aislamiento.

Estos hechos no son sorprendentes. Literatura con evidencia empírica para la región muestra que el éxito de algunos programas de fomento de medios de pago alternativos al dinero físico es menor al esperado, lo que denota la urgencia de fortalecer medidas de política pública ${ }^{[6]}$. Al respecto, persiste la necesidad de aumentar la capilaridad de aquellas empresas que atienden nichos específicos y que están focalizadas en llegar a poblaciones que hoy no acceden a servicios financieros o no se sienten atraídas por el uso de estos. Existe cierto optimismo con el rol que las FinTech (acrónimo de finance technology) pueden jugar en este sentido. Por ejemplo, BID (2019) señala que el $46 \%$ de los emprendimientos 
FinTech encuestados para el reporte afirmaron desarrollar una estrategia dirigida a consumidores y pymes sub-bancarizadas o no bancarizadas.

Para que este potencial sea viable y pueda desplegarse, es necesario contar con un entorno competitivo y regulatorio que lo habilite y facilite. Es decir, las posibilidades de expansión de los medios de pagos digitales y herramientas financieras no deberían estar limitadas por la existencia de barreras que condicionen la entrada de nuevos actores o el desarrollo de estos. En este sentido es fundamental conocer la estructura de los mercados, identificar los actores relevantes y las potenciales barreras al ingreso de nuevos operadores.

Un elemento central a la hora de analizar el desarrollo de nuevos agentes como las FinTech es el hecho de que su ingreso al mercado y su expansión dependen en gran medida de la infraestructura de pago de los incumbentes para ofrecer servicios complementarios o diferenciados (Vives 2019, OECD 2020). Es decir, para poder desarrollarse, las FinTech podrían necesitar de la infraestructura de bancos incumbentes. Estas cuestiones, junto con la regulación y la innovación en el sector, son objeto de análisis y de debate en varios foros internacionales, en particular en la OECD, que desde 2019 promueve un espacio de discusión titulado "Digital disruption in financial markets". Sin embargo, poco se ha escrito al respecto en América Latina.

El presente artículo se propone identificar barreras que pudieran estar actuando como límites al desarrollo de nuevos actores y a la innovación en el sector en la región. Se documentan y analizan medidas e intervenciones que han llevado a cabo las agencias de competencia en años recientes para disminuir esas barreras, en muchos casos motivadas por las demandas que impone la innovación tecnológica, por denuncias de jugadores entrantes o por investigaciones de mercado abiertas de oficio.

En la sección 2 se analizan las posibles estrategias de los distintos tipos de jugadores, como así también las barreras que los actores incumbentes tienen la capacidad de erigir. El estudio se lleva a cabo teniendo en cuenta la experiencia en la región, en particular, en Argentina, Brasil, Chile, México y Perú. En la sección 3 se discute por qué la irrupción digital en el sector financiero tiene implicancias muy diferentes a la irrupción en otros sectores como los de comunicaciones, contenidos, transporte de pasajeros y alojamiento turístico. Como se explica en la sección 3, estas diferencias pueden implicar la necesidad de hacer un balance entre competencia y regulación. En la sección 4 se presentan las conclusiones del trabajo.

\section{Estrategias y barreras a la entrada: situación en la región}

El sistema bancario ha dejado de ser una red de sucursales físicas para convertirse en un sector digitalizado, con alta tecnología de la información y uso de datos. Esta transformación ha supuesto el ingreso de nuevos actores, en particular, las FinTech y las 
BigTech. Las FinTech son aquellas empresas que usan tecnología innovadora en servicios financieros ${ }^{[7]}$. Las BigTech son las grandes empresas tecnológicas que están ampliando el horizonte de sus productos para proveer servicios de pagos electrónicos y financieros, y se organizan típicamente en modelos de plataformas tales como Amazon, Google o Facebook.

La llegada al sector de estos nuevos agentes implica nueva competencia para la banca tradicional que, frente a consumidores que demandan experiencias diferentes, cuentan con un exceso de capacidad de un capital que hoy ha visto reducida su utilidad.

Por otro lado, si bien las FinTech logran innovaciones que complacen a sus usuarios, tienen problemas para conseguir una escala mínima necesaria de clientes debido en gran medida a los altos costos que implica cambiar de institución financiera (World Economic Forum 2017, Vives 2019, OECD 2020). Por su parte, Vives (2019) considera que las BigTech cuentan con las ventajas de las FinTech, pero no sufren sus desventajas, están en el mejor de los mundos, porque las BigTech tienen una gran base de clientes, alta reputación, marca reconocida y bajos costos de capital; de esta manera, son las que potencialmente disponen de mayor capacidad disruptiva frente a la banca tradicional.

Dicho esto, ¿qué podemos esperar para el futuro? La banca tradicional puede asumir dos tipos de estrategias, que podrían variar entre mercados: adaptarse, colaborar y complementarse, o resistir la entrada de nuevos operadores. La Tabla 1 a continuación resume de manera esquemática las posibles maneras en que los bancos pueden llevar adelante estas estrategias.

\section{Tabla 1: Estrategias de la banca tradicional}

\begin{tabular}{|l||l|}
\hline \multirow{4}{*}{\begin{tabular}{l|l|} 
Adaptarse, colaborar y \\
complementarse
\end{tabular}} & $\begin{array}{l}\text { - Formar acuerdos de colaboración } \\
\text { - Ganancias por tasas de intercambio pagadas } \\
\text { por entrantes } \\
\text { - Nuevos clientes atraídos por los entrantes } \\
\text { - Know how en tecnología }\end{array}$ \\
\hline \hline \multirow{3}{*}{ Resistir la entrada } & $\begin{array}{l}\text { - Exclusividades } \\
\text { - Negar acceso a la infraestructura } \\
\text { - Degradar interconexión } \\
\text { - Manejo de la tasa de intercambio } \\
\text { - Venta atada } \\
\text { - Empaquetamiento }\end{array}$ \\
\hline
\end{tabular}

Fuente: Elaboración de los autores con argumentos en Barba Navaretti et al. (2017), Cade (2019), Greco y Viecens (2019), Vives (2019), OECD (2020).

Si bien las FinTech brindan servicios independientes, muchas trabajan con elementos del sistema financiero tradicional, tales como la posesión de una cuenta bancaria y el historial 
crediticio. En este sentido, la colaboración entre FinTech y bancos resulta en beneficio de ambas partes (Cámara Argentina de Fintech 2018) y puede implicar una ampliación de la cartera de clientes de los bancos, mientras que las FinTech disponen de ciertos componentes del sistema financiero indispensables para poder operar. Al mismo tiempo, la colaboración podría generarse en el desarrollo conjunto de soluciones innovadoras con mejores servicios para los clientes. Estas sinergias han llevado al surgimiento del modelo de negocio de banca abierta (open banking), que permite a los bancos proveer servicios en asociación con proveedores digitales FinTech mediante aplicaciones. Este paradigma está en el corazón de la estrategia de adaptación, cooperación y complementación entre bancos y FinTech. Los bancos aportan sus ventajas (mayor capital, marca reconocida que brinda confianza a los consumidores, mayor conocimiento de las regulaciones) y retienen como core business su licencia bancaria, su base de clientes (CRM) y la actividad de compliance (Noya 2019) ${ }^{[8]}$.

Sin embargo, no siempre la colaboración y complementación resulta ser la opción más rentable para los bancos, los cuales han sabido desplegar estrategias que implican barreras al ingreso de nuevos jugadores y a la innovación. Aquellos casos en los que estas estrategias configuren conductas anticompetitivas, ya sea porque son llevadas a cabo por un agente con posición dominante o porque involucran acuerdos anticompetitivos entre los actores incumbentes, dan lugar a la intervención de las agencias de competencia y los reguladores [9].

En años recientes varias agencias de competencia de América Latina han llevado a cabo diversos tipos de estudios, recomendaciones e investigaciones para conocer el sector y promover la competencia ${ }^{[10]}$.

En mayo de 2016 la Comisión Nacional de Defensa de la Competencia (CNDC) de Argentina abrió una investigación de mercado de tarjetas de crédito, débito y medios de pago electrónicos que concluyó con un conjunto de recomendaciones procompetitivas para el Banco Central de la República Argentina (BCRA) y con la apertura de una investigación de oficio en contra de la empresa Prisma Medios de Pago (la única licenciataria en adquirencia de Visa) y todos sus accionistas (los 14 principales bancos de Argentina y Visa Internacional $\left.{ }^{[11]}\right)$. Las teorías de daño abordadas fueron el presunto abuso de posición dominante y la realización de prácticas coordinadas (Greco y Viecens 2019). Como resultado de la investigación, los accionistas se comprometieron a vender Prisma. En enero de 2019 se vendió el 51 \% de las acciones y se transfirió control de la compañía al fondo de inversión Advent ${ }^{[12]}$.

En octubre 2019 el Conselho Administrativo de Defesa Econômica (CADE) de Brasil publicó un estudio sobre el mercado de medios de pago motivado entre otras cosas por la necesidad de avanzar en la investigación vinculada al sector frente a todos los cambios que está experimentando como resultado de las novedades tecnológicas. CADE (2019) destaca el avance de las instituciones de pago como resultado de la evolución tecnológica y señala que 
por esto mismo algunas son reconocidas como FinTech que generan presión competitiva a los bancos con una oferta de servicios de tecnología disruptiva ${ }^{[13]}$. Además, en marzo de 2019 CADE inició un proceso administrativo contra cuatro bancos por dificultar, supuestamente, el acceso a la contratación del débito automático por parte de una FinTech (Nubank) ${ }^{[14]}$. En noviembre de 2019 aplicó una medida preventiva por prácticas de venta atada a Itaú y Rede en el mercado de medios de pago ${ }^{[15]}$.

En Chile, en 2017, el Tribunal de Defensa de la Libre Competencia (TDLC) emitió una serie de recomendaciones de cambios normativos, como resultado de un requerimiento de finales de 2013 de la Fiscalía Nacional Económica (FNE). En diciembre de 2019 la Corte Suprema emitió una sentencia de gran relevancia para el sector, en busca de mayor competencia.

En Chile, donde el rol adquirente ha sido realizado por una sola empresa (Transbank) durante los últimos 15 años, en 2017, el Tribunal de Defensa de la Libre Competencia emitió una serie de recomendaciones de cambios normativos. Ello fue resultado de un requerimiento de finales de 2013 de la Fiscalía Nacional Económica, destinado a promover la competencia en la adquirencia, limitar el actuar conjunto de los bancos emisores en una única empresa y fijar tasas de intercambio máximas. A su vez, en diciembre de 2019, la Corte Suprema emitió una sentencia de gran relevancia para el sector, que obligaba a la adquirente monopólica a establecer tarifas para los comercios que fueran públicas, motivadas, objetivas, razonables, de general aplicación y no discriminatorias, con el fin promover la competencia. Ambos fallos, en los hechos, han promovido un cambio del sistema chileno hacia un modelo en el que los roles de emisor y adquirente se encuentren separados ${ }^{[16]}$.

En octubre de 2017, en México la Comisión Federal de Competencia Económica (COFECE) emitió una opinión para recomendar ajustes al proyecto inicial de la Ley para Regular las Instituciones de Tecnología Financiera (Ley Fintech) a los fines de potenciar la competencia e innovación en el mercado de los servicios financieros. En marzo de 2018, el Senado de México promulgó la Ley Fintech, y allí se incluyeron las recomendaciones recibidas en la Opinión de COFECE ${ }^{[17]}$. En México, en octubre de 2018, la Autoridad Investigadora de la Comisión Federal de Competencia Económica inició una investigación en el mercado del sistema de pagos cuyo procesamiento involucra una cámara de compensación para pagos con tarjetas, con el fin de determinar la posible existencia de barreras a la competencia y libre concurrencia, o insumos esenciales que puedan generar efectos anticompetitivos ${ }^{[18]}$. $\mathrm{Al}$ respecto, COFECE señala que de existir problemas de competencia en el mercado investigado, se estaría afectando la bancarización y la inclusión financiera. La investigación está en curso y está pendiente su resultado.

En noviembre de 2018, el Instituto Nacional de Defensa de la Competencia y de la Protección de la Propiedad Intelectual (Indecopi) de Perú anunció el inicio de un estudio de mercado para analizar las condiciones de competencia existentes en los sistemas de tarjetas de pago ${ }^{[19]}$. Al igual que la de COFECE, la investigación está en curso. 
La Tabla 2 resume las principales medidas adoptadas recientemente por las agencias de competencia en la región.

Tabla 2: Principales medidas recientes impulsadas por las agencias de competencia de la región para promover la entrada de nuevos jugadores al sistema financiero

\begin{tabular}{|c|c|c|c|c|}
\hline CNDC-Argentina & CADE-Brasil & FNE/TDLC-Chile & COFECE-México & Indecopi-Perú \\
\hline $\begin{array}{l}2016 \\
\text { Investigación de } \\
\text { mercado de } \\
\text { medios de pago } \\
\text { electrónicos e } \\
\text { investigación de } \\
\text { oficio contra } \\
\text { adquirente única } \\
\text { de Visa (Prisma) } \\
\text { y } 14 \text { bancos } \\
2017 \\
\text { Aceptación de } \\
\text { compromiso } \\
\text { estructural y } \\
\text { conductual por } \\
\text { parte de las } \\
\text { investigadas } \\
\text { 2019 } \\
\text { Desinversión de } \\
\text { los bancos en } \\
\text { Prisma } \\
\text { 2019 } \\
\text { Multiadquirencia }\end{array}$ & $\begin{array}{l}2019 \\
\text { Proceso } \\
\text { administrativo } \\
\text { contra cuatro } \\
\text { bancos por } \\
\text { supuesta } \\
\text { discriminación } \\
\text { a una FinTech } \\
\text { (Nubank) } \\
2019 \\
\text { Proceso } \\
\text { administrativo } \\
\text { y medida } \\
\text { preventiva } \\
\text { contra Itaú y } \\
\text { Rede por } \\
\text { venta atada } \\
\text { 2019 } \\
\text { Publica } \\
\text { Estudio de } \\
\text { Mercado de } \\
\text { Instrumentos } \\
\text { de Pago }\end{array}$ & $\begin{array}{l}2013 \\
\text { FNE solicita } \\
\text { cambios } \\
\text { regulatorios } \\
\text { para promover } \\
\text { competencia en } \\
\text { adquirencia de } \\
\text { tarjetas de } \\
\text { crédito } \\
2017 \\
\text { TDLC emite } \\
\text { recomendación } \\
\text { promoviendo } \\
\text { competencia y } \\
\text { cambio de } \\
\text { modelo } \\
\text { 2019 } \\
\text { Sentencia de la } \\
\text { Corte Suprema } \\
\text { avalando mayor } \\
\text { competencia y } \\
\text { cambio de } \\
\text { modelo }\end{array}$ & $\begin{array}{l}2017 \\
\text { Opinión sobre } \\
\text { proyecto de Ley } \\
\text { Fintech } \\
2018 \\
\text { Se sanciona la } \\
\text { Ley Fintech con } \\
\text { recomendaciones } \\
\text { de COFECE } \\
2018 \\
\text { Investigación en } \\
\text { el mercado del } \\
\text { sistema de pagos } \\
\text { cuyo } \\
\text { procesamiento } \\
\text { involucra una } \\
\text { cámara de } \\
\text { compensación } \\
\text { para pagos con } \\
\text { tarjetas }\end{array}$ & $\begin{array}{l}2018 \\
\text { Inicio de un } \\
\text { estudio de } \\
\text { mercado para } \\
\text { analizar las } \\
\text { condiciones } \\
\text { de } \\
\text { competencia } \\
\text { existentes en } \\
\text { los sistemas } \\
\text { de tarjetas de } \\
\text { pago }\end{array}$ \\
\hline
\end{tabular}

Fuente: Elaboración de los autores con información pública.

La Tabla 2 informa la intensa actividad de las agencias de competencia de América Latina en relación con el sector de medios de pago electrónicos y digitales. Al respecto, como se observa en la tabla, las agencias cuentan con diversas herramientas para utilizar en función de la situación que se busca abordar. Las investigaciones de mercado analizan las condiciones de competencia e identifican las potenciales barreras que puedan existir. Estas investigaciones pueden derivar en investigaciones de oficio por prácticas anticompetitivas de algún agente específico o en recomendaciones procompetitivas para reguladores, como los bancos centrales. Las investigaciones por posibles conductas anticompetitivas pueden surgir también como resultado de denuncias presentadas por competidores o agentes afectados por tales prácticas.

Del análisis llevado a cabo, se deducen ciertos rasgos en común en relación con la actividad desplegada por las agencias de la región. Algunos procesos se iniciaron con estudios e 
investigaciones de mercado que han derivado en recomendaciones procompetitivas (Chile y Argentina), mientras que las de México y Perú aún se encuentran en curso. En algunos casos como el de Argentina, han implicado también la apertura de una investigación de oficio por conductas anticompetitivas. Por su parte, en Brasil, CADE ha abierto investigaciones contra bancos a raíz de denuncias de competidores o entrantes (FinTech).

De los estudios e investigaciones relevados en los diferentes países de la región, surge un conjunto de potenciales barreras que las agencias han buscado eliminar. A continuación analizamos los principales aspectos que resultan relevantes para la evaluación de las condiciones de competencia en medios de pago electrónico. En primer lugar, se discuten algunas características particulares de estos mercados y su implicancia desde el punto de vista de defensa de la competencia. Luego se describen las potenciales barreras para la entrada de nuevos jugadores que han sido identificadas.

\subsection{La evaluación de las condiciones de competencia en presencia de plataformas multilaterales $^{[20]}$}

La metodología usual para evaluar las condiciones de competencia en los mercados se inicia con la definición de los mercados relevantes para luego analizar la existencia de poder de mercado de alguno de sus actores. La aplicación de esta metodología a los mercados de plataformas multilaterales presenta algunos dilemas que han sido abordados por la literatura económica desde el trabajo seminal de Rochet y Tirole (2003) y Armstrong (2006).

Los mercados de tarjetas de crédito y medios de pago electrónico pertenecen a la categoría de plataformas que conectan distintos tipos de clientes y que se han dado en llamar plataformas bilaterales o multilaterales. Una definición aplicable a este tipo de mercados puede ser la que provee el estudio de la OECD (2018): un mercado en el que una firma actúa como una plataforma y vende diferentes productos a diferentes grupos de clientes, reconociendo que la demanda de un grupo depende de la demanda de otro(s) grupo(s).

Una de las características distintivas de estos mercados es la relación entre la demanda de los distintos grupos de usuarios de la plataforma (vgr. los distintos lados de la plataforma), que da lugar a efectos de red indirectos. Estos efectos constituyen externalidades, ya que un mayor número de usuarios de un lado de la plataforma la hacen más atractiva para los usuarios del otro lado. Cuando estos efectos son significativos, los precios que la plataforma carga a los usuarios de un lado afectan la demanda del otro lado, y dan lugar a reacciones en la demanda del primer grupo.

La literatura y la jurisprudencia internacional en organización industrial y defensa de la competencia coinciden en que una adecuada evaluación de estos mercados requiere considerar en el análisis la interrelación entre ambos lados del mercado y algunos autores sostienen que para casos que involucran plataformas de transacciones, como es el caso de los mercados de tarjetas de crédito y medios de pago electrónico, debería definirse un único mercado relevante que incluya ambos lados de la plataforma. Este criterio ha sido el 
adoptado en el reciente fallo de la Corte Suprema de Estados Unidos en el caso AMEX (por una mayoría de 5 contra 4), que consideró el mercado relevante de transacciones con tarjeta de crédito (SCUS 2018) ${ }^{[21]}$.

El criterio que se ha adoptado en la mayor parte de los casos en la región ha sido diferente y por razones que lo justifican. El modelo de negocios con el que las marcas más utilizadas en América Latina (Visa y Mastercard) operan en el mundo es diferente al de AMEX, ya que incluye nuevos actores (los adquirentes y los emisores) y un precio adicional, la tasa de intercambio. Esta tasa es el pago que los adquirentes (empresas que ofrecen las tarjetas de crédito a los comercios) realizan a los emisores (los bancos que entregan tarjetas de crédito entre sus clientes). En el caso de Argentina, Prisma (la única adquirente de Visa) fijaba una alta tasa de intercambio, que cobraban sus propios accionistas (los bancos), y que dejaba el negocio de adquirencia sin rentabilidad y sin incentivos para potenciales nuevos adquirentes. Prisma contaba además con el negocio de procesamiento de Visa, lo que podía contribuir a que los bancos tuvieran menos incentivos para emitir y promover el uso de otras marcas de tarjetas de crédito. Finalmente, el dominio de Prisma de la infraestructura y servicios asociados al procesamiento afectaba la posibilidad de ingreso y desarrollo de las FinTech, ya que generaba las condiciones y los incentivos para que los bancos incumbentes y Prisma dificultaran la interconexión y el acceso de las FinTech a los datos y servicios necesarios para desarrollar sus servicios innovadores.

En este marco, con el fin de poder identificar el origen de las posibles restricciones a la competencia y los remedios necesarios para eliminar las barreras de entrada, resultaba necesario analizar cada uno de los eslabones de la cadena de valor. Una definición de mercado único de transacciones lo hubiera impedido. Para desarmar las barreras a la competencia y al ingreso de nuevos jugadores, había que promover la competencia en la adquirencia y es por eso que se definió un mercado relevante para esta actividad (además de un mercado de emisores y de transacciones).

Por su parte, el CADE de Brasil ha considerado los mercados de emisión, adquirencia y el de la marca. En particular, ha advertido que, dada la característica de plataforma de múltiples lados del sector de medios de pagos y la presencia de externalidades de redes, resulta necesario considerar a todos estos agentes en el análisis, pero ha definido cada uno como un mercado relevante diferente (CADE 2019). Además, en algunos de los casos tratados en la agencia, si bien se identificó a la adquirencia como un mercado relevante, se destacó la interdependencia entre los diferentes agentes, emisores, marca y adquirentes (CADE 2019).

Nótese que la adopción de este enfoque no implica una contradicción con el fallo de la Corte Suprema de Estados Unidos en AMEX, básicamente porque los casos no son comparables ${ }^{[22]}$. Además, la definición de mercados relevantes utilizada por CADE y la CNDC no es un precedente que implique que no pueda utilizarse una definición de mercado diferente, por ejemplo, en una investigación de prácticas comerciales por parte de las marcas de tarjetas 
de crédito.

Una mención aparte merece el caso de Chile. La FNE en el requerimiento de 2013 al TDLC utilizó también este enfoque en su análisis, con una definición explícita del mercado de adquirencia que sustenta las recomendaciones (FNE 2013). El TDLC, sin realizar una definición explícita del mercado relevante de adquirencia, adopta implícitamente este enfoque en su fundamentación de las recomendaciones para eliminar barreras de entrada en esa actividad (TDLC 2017) ${ }^{[23]}$. Sin embargo, una sentencia de diciembre de 2019 de la Corte Suprema definió el mercado relevante de las transacciones efectuadas utilizando como medio de pago tarjetas de crédito o débito (Corte Suprema de Chile 2019). En esa misma sentencia la Corte Suprema llamó a las autoridades a regular la tasa de intercambio. Obsérvese que existe una tensión entre la definición de mercado relevante adoptada y esta recomendación regulatoria. La tasa de intercambio es el precio que conecta ambos lados del mercado (es la tasa que el adquirente paga al emisor por cada transacción) y que, por ende, internaliza los efectos indirectos de red existentes. Regular esa tasa lleva a perder dicha internalización e implica cierta contradicción con la definición de mercado adoptada, aunque resulta coherente con el diagnóstico de falta de competencia en adquirencia (véanse los apartados 2.2.3 y 2.2.4 en la próxima sección).

\subsection{Barreras al ingreso de nuevos jugadores}

En línea con las Tablas 1 y 2, las principales características de las estructuras de mercado y las conductas de las firmas que se han identificado como potenciales barreras a la entrada en los mercados de medios de pago electrónico y que han sido objeto de investigación y análisis por parte de las autoridades de competencia en la región se reúnen en cuatro grupos que se describen a continuación.

\subsubsection{Exclusividades de las marcas importantes con algunos bancos}

La existencia de condiciones de exclusividad de alguna marca dominante puede conformarse como una barrera a la entrada y al crecimiento de las FinTech. En Brasil la existencia de exclusividades de VisaNet (hoy Cielo) con la marca Visa y Redecard (hoy Rede) con Mastercard dio lugar a varios casos y denuncias en la agencia de competencia ${ }^{\text {[24] }}$. Como consecuencia, en diciembre de 2009, Visa y VisaNet dieron de baja el contrato de exclusividad con un acuerdo de compromiso de cesación de prácticas (cessação de prática) que impulsó la multiadquirencia en Brasil ${ }^{[25]}$. En julio de 2010 tanto VisaNet como Redecard comenzaron a ser adquirentes de marcas alternativas. El proceso se consolida en 2013 con la entrada en vigor de la Ley 12.865 que promueve la interoperabilidad y el acceso no discriminatorio a los servicios de pago (CADE 2019).

En Argentina, la empresa Prisma contaba con una exclusividad de hecho en la adquirencia de Visa y la empresa First Data, con la exclusividad de Mastercard. Como resultado de la investigación y recomendaciones de la CNDC y de las medidas adoptadas por el BCRA, en Argentina hay multiadquirencia desde enero de 2019 (Greco y Viecens 2019). 
En Chile, tanto la FNE como el TDLC han señalado su preocupación por la exclusividad de Transbank para la adquirencia de tarjetas de crédito y la necesidad de generar competencia en este mercado (FNE 2013 y TDLC 2017). En abril de 2019 el Gobierno impulsó cambios normativos para eliminar la exclusividad de Transbank como adquirente ${ }^{[26]}$. En diciembre de 2019 la Corte Suprema avaló las recomendaciones de apertura a la competencia (Corte Suprema de Chile 2019).

En Perú, este es uno de los potenciales problemas destacados en el estudio preliminar realizado por Indecopi para fundamentar la apertura del estudio de mercado, dado que VisaNet es la única adquirente de Visa y PMP la única adquirente de Mastercard ${ }^{[27]}$.

En México, en 2014, la Ley para la Transparencia y Ordenamiento de los Servicios Financieros prohibió las exclusividades para diversos servicios financieros, e incluyó las referidas a redes de medios de disposición ${ }^{[28]}$ y las establecidas en los contratos con comercios, emisores y adquirientes. Además, la ley indica que las autoridades regulatorias deben establecer los términos y las condiciones para la prestación de los servicios de modo que no constituyan barreras a la entrada y, en particular, deben evitar la exclusividad para la utilización algún tipo de red (COFECE 2014).

Se concluye entonces que, si bien las relaciones de exclusividad han sido una característica relevante en estos mercados, la evidencia en la región muestra una tendencia a la desaparición, lo que es congruente con la promoción de competencia en el sector.

\subsubsection{Integración vertical sin condiciones de acceso a la infraestructura}

La presencia de estructuras verticales cerradas conformadas por un grupo de incumbentes son barreras para el desarrollo de jugadores BigTech y FinTech en estos mercados, en particular en el canal de comercio electrónico.

El caso argentino se caracterizaba por el rol preponderante de la principal incumbente -Prisma- que era la única empresa adquirente de la marca Visa en Argentina, estaba presente en todos los eslabones de la cadena de servicios de medios de pago electrónicos, en ambos canales (presencial y electrónico), y sus accionistas eran 14 bancos de la Argentina. Un elemento fundamental fue el hecho de que la integración de Prisma la convertía en la única opción en algunos eslabones de la cadena de valor. Por ejemplo, los entrantes que quisieran ofrecer opciones de medios de pago e incluir en su portafolio la marca Visa debían recurrir a Prisma y no tenían opciones. Es decir, Prisma contaba con una infraestructura esencial en Argentina para todo comercio presencial u online, y para todo potencial oferente de servicios de pago electrónico o facilitador de pagos, que quisiera operar con Visa, la marca más usada en el país (Greco y Viecens 2019). A los fines de abordar este problema, en el Compromiso asumido por Prisma ante la Autoridad de Defensa de la Competencia, se incluyeron un conjunto de condiciones conductuales de "acceso abierto" que aseguraran la provisión a terceros de los servicios de procesamiento prestados por Prisma en condiciones no discriminatorias, como así también cualquiera de los servicios 
prestados por Prisma en el mercado de pagos electrónicos.

En Chile, la estructura de mercado ha sido aún más cerrada que la de Argentina. En particular, los bancos tenían delegada de manera conjunta la adquirencia en Transbank (sociedad de propiedad conjunta de 13 bancos) y prácticamente toda la adquirencia pasaba por Transbank (TDLC 2017). En este sentido, las Recomendaciones del TDLC incluyeron obligaciones de acceso abierto a las redes de procesamiento, como así también regular las condiciones bajo las cuales los proveedores de red contrataran con los potenciales proveedores de servicios de adquirencia (TDLC 2017).

En México, como ya se mencionó, se promulgó la Ley Fintech. La ley incluye recomendaciones que realizó COFECE: i) el establecimiento de reglas para clarificar la propiedad y acceso a los datos; ii) garantizar la no discriminación por parte de instituciones financieras más grandes hacia las FinTech; y iii) eliminar la infraestructura restrictiva o tecnológica que impiden la entrada de participantes.

En Perú, el Indecopi destaca que la integración vertical entre adquirencia exclusiva y procesamiento genera duplicación de costos para los comercios, tales como el alquiler mensual del equipo POS en sus modalidades convencional o inalámbrico ${ }^{[29]}$.

El acceso a la infraestructura de los bancos se incluye entre las principales demandas realizadas por las FinTech para poder competir ${ }^{[30]}$. En este sentido, el caso Prisma de Argentina, la Ley para Regular las Instituciones de Tecnología Financiera en México y el proceso que experimenta Chile en estos momentos dan cuenta de una clara intención por parte de los Gobiernos de romper viejas estructuras que pudieran estar entorpeciendo el desarrollo de entrantes innovadores en el sector.

\subsubsection{Una alta tasa de intercambio que genera baja rentabilidad en el servicio de adquirencia}

El análisis de la tasa de intercambio y su tratamiento regulatorio -si debe ser regulada o dejarse librada al mercado- es uno de los puntos más controversiales en la literatura y en la experiencia internacional. Como ya se explicó, los mercados de pagos electrónicos son mercados de dos lados y la tasa de intercambio define y altera la estructura de precios relativa entre estos dos lados (Rochet y Tirole 2002).

En Argentina, la CNDC entendió que la alta tasa de intercambio configuraba una barrera a la entrada de nuevos adquirentes. El arancel que pagan los comercios como porcentaje de las ventas remunera la adquirencia y la emisión de tarjetas de crédito, por lo cual es igual a la suma de la tasa de intercambio que cobran los emisores y el margen de adquirencia que remunera dicha actividad. Dado que las tasas de intercambio eran cercanas al valor máximo del arancel, el margen de adquirencia era muy bajo y no había incentivos económicos para la entrada de nuevos adquirentes, por lo que la CNDC recomendó que sean reguladas ${ }^{\text {[31] }}$. En marzo de 2017 el Banco Central dispuso la regulación de tasas de intercambio máximas para tarjetas de crédito, débito y compra con un cronograma descendente ${ }^{[32]}$. 
En Brasil la tasa de intercambio para operaciones con tarjeta de débito está regulada desde $2018^{[33]}$. En Chile, el TDLC, siguiendo la propuesta de la FNE, incluyó entre sus recomendaciones la de regular la tasa de intercambio para fomentar la actividad en la adquirencia (TDLC 2017). En diciembre de 2019, la Corte Suprema instó a las autoridades competentes para que consideren la regulación de las tasas de intercambio (Corte Suprema de Chile 2019) ${ }^{[34]}$.

En relación con la tasa de intercambio, no hay consenso en la literatura a nivel internacional sobre si debe o no ser regulada. De la revisión llevada a cabo, se deduce que en la región la experiencia es diversa, aunque en años recientes se observa una tendencia hacia la regulación de dicha tasa.

\subsubsection{Empresa adquirente con posición dominante y conformada por bancos emisores}

La posición dominante en la adquirencia de una empresa cuyos dueños son los bancos emisores tiene el potencial de ocasionar dos tipos de problemas. Por un lado, genera incentivos para erigir barreras de entrada al mercado de medios de pago electrónico a jugadores no integrados. Por otro lado, facilita intercambios de información estratégica entre competidores que afecten la competencia en mercados adyacentes, tales como el de financiamiento al consumo.

Ya se ha mencionado que esta ha sido la situación en Argentina. La investigación identificó que la composición accionaria de Prisma la constituía en un potencial vehículo para la coordinación de estrategias comerciales entre los bancos. Es por esto que en septiembre de 2017 se instrumentó un remedio estructural, mediante un Compromiso que incluyó la venta de las acciones de Prisma por parte de los bancos, el primero en un caso de investigación por presuntas conductas anticompetitivas en Argentina ${ }^{[35]}$.

En Chile, las recomendaciones del TDLC incluyeron la prohibición a bancos emisores de actuar conjuntamente en la adquirencia. Recuérdese que Transbank -prácticamente único adquirente en el momento de la recomendación- era propiedad de 13 bancos emisores. En abril de 2019, el Ministerio de Hacienda anunció un conjunto de medidas para promover la competencia en el mercado de tarjetas de crédito y débito que incluyen impulsar un modelo en el que adquirencia y emisión operen de manera separada ${ }^{[36]}$. Además, el Ministerio argumentó que la estructura actual del mercado implica barreras a la entrada para nuevos jugadores ${ }^{[37]}$.

En Perú, VisaNet (adquirente de Visa) tiene como accionistas a los principales emisores del país (BCP, BBVA, Scotiabank e Interbank). Además, Scotiabank e Interbank son los accionistas de PMP (adquirente de Mastercard) ${ }^{[38]}$.

Se deduce entonces, retomando la discusión de la Sección 2.1, que la experiencia de América Latina nos permite explicar una situación en la cual suele resultar conveniente definir el mercado de adquirencia de manera separada y regular la tasa de intercambio. Es 
decir, frente a un mercado cerrado como los de Argentina y Chile (donde los emisores son dueños de un adquirente con posición dominante), las altas tasas de adquirencia reducen el margen de rentabilidad en la adquirencia y actúan como barreras a la entrada de nuevos jugadores. Sin intervención, esa estructura se mantiene estable dado que para los emisores son rentables las altas tasas ${ }^{[39]}$. La situación puede ser diferente una vez que ya hay competencia en la adquirencia o cuando se investiguen prácticas comerciales por parte de las marcas de tarjetas de crédito en el contexto de un mercado maduro y competitivo.

\title{
3. ¿Por qué el impacto de la digitalización y la introducción de las nuevas tecnologías en el sector financiero es diferente?
}

\begin{abstract}
A lo largo de la historia, las crisis financieras han sido el origen o el mecanismo de transmisión de las principales recesiones y depresiones de la economía internacional. Por ende, el sector financiero tiene la capacidad de afectar a toda la economía a través de las propias crisis. Esta particularidad ha implicado que la regulación financiera incluya entre sus objetivos principales el de proteger la estabilidad del sistema. Ante cambios tecnológicos disruptivos, este objetivo podría entrar en conflicto con los objetivos de defensa de la competencia cuando promover la competencia pueda poner en riesgo la estabilidad financiera. Este conflicto no se presenta en otros mercados en los cuales las plataformas digitales han irrumpido como jugadores sustanciales ${ }^{[40]}$.
\end{abstract}

Un antecedente relevante que puso en evidencia este dilema es el de los años 80, cuando se redujo el peso de la estabilidad financiera entre los objetivos regulatorios, lo que dio lugar a una reducción de las barreras de entrada y la eliminación de normas que protegían a los bancos de la competencia (Bofondi y Gobbi 2017). El surgimiento de innovaciones tecnológicas, que permitieron el desarrollo de fuentes de competencia para los bancos como los money market funds, que presionaron sobre su rentabilidad en un contexto de desregulación gradual, llevaron a la expansión del shadow banking y fueron el origen de la crisis de $2008^{[41]}$.

La actual situación de transformación digital disruptiva vuelve a resaltar la relevancia de este dilema entre competencia y estabilidad financiera, y la necesidad de coordinación entre las políticas de regulación financiera y de defensa de la competencia. El desafío planteado en el contexto actual con nuevos actores BigTech y FinTech consiste en maximizar los beneficios de la competencia y la innovación sin comprometer la estabilidad financiera. Un desafío de esta magnitud no ha sido expuesto, por ejemplo, en el caso de la irrupción de las over-the-top (OTT) en los mercados de comunicaciones y contenidos, o con el ingreso de Uber en el mercado de transporte de pasajeros, por mencionar solo algunos ejemplos.

Los riesgos para la estabilidad financiera reconocen distintas fuentes (Vives 2019, OECD 2020, Bofondi y Gobbi 2017, Barba Navaretti et al. 2017). En primer lugar, si la entrada de 
las FinTech y las BigTech reduce la rentabilidad de los incumbentes, ello aumenta los incentivos para que estos tomen riesgos excesivos para compensar el impacto. Al mismo tiempo, si las regulaciones financieras prudenciales fueran estrictas e impidieran el apalancamiento excesivo, esto podría incrementar la actividad de shadow banking por fuera del perímetro regulatorio.

El impacto de la disrupción digital puede generar asimetrías de información que impliquen nuevas fuentes de riesgo sistémico. Por un lado, pueden plantearse problemas de moral hazard ${ }^{[42]}$ cuando las plataformas tienen poca participación en los créditos que contribuyen a originar, ya que el incentivo lleva a incrementar el volumen para obtener comisiones (fees) lo cual actúa en detrimento de la calidad crediticia. Por otro lado, pueden surgir problemas de selección adversa si las BigTech aprovechan su capacidad en la acumulación y análisis de datos para seleccionar los préstamos más rentables y de mejor calidad (cream skimming), dejando a los bancos tradicionales con créditos de menor calidad y mayor riesgo.

Otras fuentes de riesgo para la estabilidad del sistema financiero pueden provenir del desarrollo de sistemas de pago paralelos sin adecuada supervisión de los bancos centrales, ciberataques o fallas operativas en casos de alta provisión de servicios a terceros en la nube (tales como almacenamiento, transmisión o análisis de datos) y la emergencia de grandes fondos money market online que no cuenten con seguros. Finalmente, un ingreso de las BigTech en la actividad bancaria podría implicar una "contaminación" de esta actividad por la actividad no bancaria.

Por su parte, De la Mano y Padilla (2019) sostienen que las BigTech podrían monopolizar el mercado de préstamos para consumidores y pymes, lo que podría dañar la competencia y generar inestabilidad financiera.

Esto lleva a que hoy la discusión internacional esté enfocada en determinar el equilibrio regulatorio que permita balancear el trade-off entre competencia y estabilidad financiera frente al ingreso de nuevos actores ${ }^{[43]}$. En América Latina, la valoración del trade-off entre competencia y estabilidad financiera es relevante de cara al futuro, tanto en el diseño regulatorio como en la forma en que se desenvuelva la interacción entre FinTech, BigTech y bancos. Sin embargo, nótese que la eliminación de restricciones y barreras de entrada en los mercados de medios electrónicos de pago que surgen de las diversas investigaciones iniciadas por las agencias de competencia de la región, y que hemos analizado en este artículo, no conllevan un conflicto con los objetivos de estabilidad financiera. En este sentido, pueden ser entendidas como una fase previa, y como una condición necesaria, para que el trade-off se manifieste. El surgimiento de nuevos adquirentes de tarjetas de crédito que brinden opciones a los comercios, y la aparición y expansión de nuevas opciones de medios de pago digitales, no implica un riesgo para la estabilidad del sistema financiero. Por el contrario, son herramientas que permiten incrementar la inclusión financiera, al ampliar la base de clientes del sistema y favorecer el acceso a medios de pagos innovadores por parte de comercios que, por ejemplo, solo aceptan pagos con dinero físico. 
Finalmente, en América Latina es relevante promover la coordinación de políticas públicas de regulación financiera y defensa de la competencia no solo para asegurar la estabilidad financiera, sino, en especial, para evitar que con el argumento de protegerla se limite innecesariamente la competencia y la innovación.

\section{Discusión final y conclusiones}

El sistema financiero está transitando a nivel internacional importantes cambios tecnológicos disruptivos en el que nuevos jugadores, las FinTech, son los protagonistas. Los cambios no solo implican el ingreso de nuevos competidores en la provisión de servicios financieros existentes, sino también innovaciones que involucran el surgimiento de nuevos servicios y cambios en la forma en que se relacionan los prestadores de servicios con los consumidores. Esta transformación ha dado lugar a distintas estrategias por parte de los principales agentes económicos, los bancos o incumbentes. Las estrategias desplegadas por las firmas tecnológicas difieren en función de sus propias características. En este contexto, la regulación y las condiciones de competencia en el sector, y en particular su impacto en la innovación, están siendo objeto de análisis y de debate en varios foros internacionales.

Este proceso se manifiesta en América Latina de una forma particular. Por un lado, este fenómeno disruptivo de nuevos jugadores en servicios financieros en general y en medios de pago en particular está creciendo en países emergentes, incluidos los de América Latina ${ }^{[44]}$. Ello se explica en parte porque ha surgido una nueva oferta de servicios focalizada en usuarios no bancarizados. Por otro lado, diversas agencias de competencia de la región han iniciado investigaciones de los mercados de medios de pago electrónico a los fines de asegurar el desarrollo competitivo del ingreso de los operadores innovadores. Estas investigaciones exhiben rasgos comunes en cuanto a la estructura del mercado, a las condiciones de competencia y al tipo de barreras a la entrada de nuevos jugadores.

En este artículo hemos analizado la experiencia reciente de un conjunto de agencias de la región, en particular las de Argentina, Brasil, Chile, México y Perú. El análisis comparativo es útil para reguladores y agencias de competencia de todos los países de América Latina que se enfrentan hoy al desafío de promover la competencia y la innovación en mercados complejos y de alta relevancia por los efectos que pueden tener sobre toda la economía. En este sentido, una de las características distintivas de la digitalización y la introducción de innovaciones tecnológicas en el sector financiero es que, si bien traen competencia y beneficios para los consumidores, también pueden ser una fuente de riesgo para la estabilidad del sistema financiero. Como consecuencia, pueden surgir conflictos de objetivos entre las políticas de defensa de la competencia y las de regulación financiera, si surge un trade-off entre promover el ingreso ilimitado de oferentes y evitar un incremento del riesgo sistémico más allá de niveles razonables. Sin embargo, del análisis realizado en este trabajo se deduce que la remoción de los principales obstáculos y barreras a la innovación y la competencia que surgen de las investigaciones de las agencias de competencia de la región 
consideradas no despiertan un conflicto de objetivos con la regulación financiera. Por el contrario, constituyen una condición para que puedan surgir y desarrollarse innovaciones disruptivas. Es decir, la remoción de las barreras analizadas es una condición para que el trade-off se manifieste, una vez que las innovaciones se desarrollen. Por consiguiente, el objetivo de resguardar la estabilidad financiera no sería un argumento válido para los incumbentes en un intento de evitar la eliminación de barreras de entrada como las que se identificaron en este trabajo.

Las agencias de competencia y los reguladores financieros se enfrentan hoy en América Latina al doble desafío de promover los cambios normativos y de las estructuras de mercado que favorezcan el surgimiento y desarrollo de las FinTech, generando entornos regulatorios que eviten poner en riesgo la estabilidad del sistema financiero en el marco de un mercado competitivo. Al mismo tiempo, esto representa una gran oportunidad para los países de la región, por el potencial que implica como mecanismo acelerador de la inclusión financiera.

\section{Referencias bibliográficas}

Armstrong, Mark. 2006. Competition in Two-Sided Markets. RAND Journal of Economics 37, pp. 668-691.

Barba Navaretti, Giorgo, Giacomo Calzolari y Alberto Pozzolo. 2017. Fintech and Banks: Friends or Foes?. European Economy. Banks, Regulation, and the Real Sector, pp. 9-31.

BID. 2019. Informe Fintech en América Latina 2018: crecimiento y consolidación.

Bofondi, Marcello y Giorgo Gobbi. 2017. The Big Promise of Fintech, Fintech and Banks: Friends or Foes?. European Economy. Banks, Regulation, and the Real Sector, pp. 107-119.

CADE. 2019. Cadernos do Cade - Mercados de instrumentos de pagamento.

http://www.cade.gov.br/noticias/cade-divulga-estudo-sobre-mercado-de-instrumentos-de-pag amento.

Cámara Argentina de Fintech. 2018. Informe Ecosistema Fintech Argentino.

https://camarafintech.com.ar/wp-content/uploads/2020/01/BID-C\%C3\%A1mara-Argentina-de -Fintech-Accenture.pdf.

Carballo, Ignacio E. y Mateo Bartoloni. 2019. Pagos digitales para la inclusión financiera de poblaciones vulnerables en Argentina: un estudio empírico en comerciantes del Barrio Padre Carlos Múgica (ex Villa 31-31 bis) de la Ciudad Autónoma de Buenos Aires. http://www.economicas.uba.ar/wp-content/uploads/2019/12/CARBALLO-y-BARTOLINI-2019Pagos-Digitales-en-el-Barrio-31.pdf.

COFECE. 2014. Trabajo de investigación y recomendaciones sobre las condiciones de competencia en el sector financiero y sus mercados, México.

https://www.cofece.mx/cofece/images/Estudios/ResumenEjecutivo26-09-14.pdf\#pdf. 
Corte Suprema de Chile. 2019. Sentencia del 27 de diciembre de 2019.

https://www.fne.gob.cl/wp-content/uploads/2019/12/24.828-18_Libre-Competencia-002-Cort e-Suprema.pdf

De la Mano, Miguel y Jorge Padilla. 2018. Big Tech banking. Journal of Competition Law \& Economics, 14, pp.494-526.

Evans, David y Richard Schmalensee. 2019. The Role of Market Definition in Assessing AntiCompetitive Harm in Ohio v. American Express. https://ssrn.com/abstract=3401325 o http://dx.doi.org/10.2139/ssrn.3401325.

Financial Conduct Authority. 2015. Regulatory sandbox.

https://www.fca.org.uk/publication/research/regulatory-sandbox.pdf

FNE. 2013. Solicitud de la FNE sobre modificación del Dictamen N 757 de la H. Comisión Preventiva Central. https://www.fne.gob.cl/wp-content/uploads/2013/12/cons_01_2013.pdf

Greco, Esteban y Fernanda Viecens. 2020. ¿Por qué el caso de tarjetas de crédito en Argentina es diferente al de AMEX en Estados Unidos? Opinión técnica N 8, Programa Libre Competencia de la Pontificia Universidad Católica de Chile.

Greco, Esteban y Fernanda Viecens. 2019. Innovación y disrupción digital en los mercados de medio de pagos: el caso de defensa de la competencia en Argentina. Documento de trabajo CETyS, UdeSA. http://dx.doi.org/10.2139/ssrn.3401325

Katz, Michael. 2019. Platform Economics and Antitrust Enforcement: A Little Knowledge is a Dangerous Thing. Journal of Economics \& Management Strategy 28, 1, pp. 138-152.

Katz, Michael y Jonathan Sallet. 2018. Multisided platforms and antitrust enforcement. Yale Law Journal 127, 7, pp. 2142-2175.

Mansilla Fernández, José Manuel. 2017. Institutions, Fintech and Banks: Friends or Foes?. European Economy. Banks, Regulation, and the Real Sector, pp. 43-50.

Noya, Eloi. 2019. The Fintech Revolution: Who Are The New Competitors In Banking?.

Forbes.

https://www.forbes.com/sites/esade/2019/07/30/the-fintech-revolution-who-are-the-new-com petitors-in-banking/\#80ebeb116174.

OECD. 2020. Digital disruption in banking and its impact on competition.

http://www.oecd.org/daf/competition/digital-disruption-in-financial-markets.htm.

OECD. 2018. Rethinking antitrust tools for multi sided platforms.

http://www.oecd.org/competition/rethinking-antitrust-tools-for-multi-sided-platforms.htm.

Rochet, Jean-Charles y Jean Tirole. 2003. Platform competition in two-sided markets. Journal of the European Economic Association 1, 4, pp. 990-1029. 
Rochet, Jean-Charles y Jean Tirole. 2002. Cooperation among Competitors: Some Economics of Payment Card Associations, The RAND Journal of Economics 33, 4, pp. 549-570.

SCUS. 2018. Supreme Court of the United States, 16-1454 Ohio v. American Express Co. (06/25/2018).

TDLC. 2017. Proposición de modificación Normativa $N^{\circ}$ 19/2017 sobre servicios asociados a la utilización de tarjetas de crédito y débito de aceptación universal. Rol ERN N²0-2014. https://www.tdlc.cl/nuevo_tdlc/wp-content/uploads/2017/01/Proposicion_19_2017.pdf.

Vives, Xavier. 2019. Digital Disruption in Banking, OECD, background for Item 5 at the 131st Meeting of the Competition Committee on 5-7 June 2019. https://one.oecd.org/document/DAF/COMP(2019)1/en/pdf.

Vives, Xavier. 2017. The Impact of Fintech on Banking, European Economy. Banks, Regulation, and the Real Sector, 2, pp. 97-106.

World Economic Forum. 2017. Beyond FinTech: a pragmatic assessment of disruptive potential in financial services. Future of Financial Services Series.

\title{
Agradecimientos
}

Todo lo que aquí se detalla es público y se deduce de los dictámenes, resoluciones y disposiciones públicos que se citan a lo largo del artículo. Los autores han sido presidente y vocal de la Comisión Nacional de Defensa de la Competencia durante el periodo (2016-2020) en que la agencia llevó a cabo el caso antitrust de mayor impacto en el mercado de medios de pagos electrónicos en Argentina, como así también colaboraciones con el Banco Central para el impulso de medidas tendientes a la apertura y al desarrollo de medios de pagos innovadores. Se agradecen los comentarios de Felipe Irarrázabal, Cristián Reyes y dos evaluadores anónimos. El artículo es de exclusiva responsabilidad de los autores.

\section{Biografía de los autores}

\author{
Esteban Manuel Greco: Consultor internacional experto en competencia y \\ regulación. Ejerce la docencia en UBA y UTDT.
}

María Fernanda Viecens: Investigadora de CONICET con sede en el CETYS (Centro de Tecnología y Sociedad) de la Universidad de San Andrés (UdeSA), donde también ejerce la docencia. 


\section{Notas}

La experiencia de estar en casa encerrados se torna más agradable de esta manera, cuando por ejemplo se puede acceder a servicios online tales como clases de yoga, baile, cocina y un sinfín de actividades que las familias han elegido consumir desde sus hogares en estas circunstancias.

El código QR (Quick Response) es la evolución del código de barras y permite acceder de manera rápida a cierta información. Tiene un amplio abanico de funcionalidades. Una de ellas es que el código QR se integra con las billeteras electrónicas y se utiliza para realizar pagos presenciales con el teléfono móvil.

Véase: https://www.ambito.com/economia/billeteras/se-duplico-el-uso-billeteras-electronicascomomedio-pago-el-aislamiento-n5095115; https://www.perfil.com/noticias/bloomberg/bcfintech- deargentina-crecen-en-medio-de-la-cuarentena.phtml.

La pandemia puso en evidencia un potencial mundo virtual global para una gran cantidad de productos básicos, como así también para otros que generan nuevas experiencias atractivas en la situación de aislamiento (https://www.clarin.com/gourmet/venta-vino-online-catas-virtualesreinventan- bodegascuarentena_0_j_i8YsWLs.html).

En Argentina, largas filas de espera en las puertas de los bancos para el retiro de dinero de jubilaciones, o de subsidios a sectores desfavorecidos, se transformaron en pesadillas para adultos mayores y poblaciones de bajos recursos acostumbradas a utilizar dinero físico a la hora de realizar pagos https://www.infobae.com/sociedad/2020/04/03/largas-filas-en-laspuertas- de-los-bancos-para-cobrarjubilaciones-y-auh/; https://www.clarin.com/politica/ coronavirus-argentina-reabren-bancos-largas-colaspago-jubilaciones-planes-sociales_0_1Lj_ Mgz5R.html. Una situación similar surge con las ayudas estatales generadas específicamente a raíz de la pandemia, ya que las poblaciones beneficiarias de los programas sociales suelen no estar bancarizadas ni habituadas al uso de medios digitales para sus transacciones.

Carballo y Bartoloni (2019) documentan los resultados de 104 entrevistas a comerciantes de un asentamiento urbano de población vulnerable en la Ciudad Autónoma de Buenos Aires (Argentina). El estudio informa que, luego de algunos meses de intervenciones del Estado promoviendo medidas de bancarización y uso de medios de pagos electrónicos entre los comerciantes y las personas que viven en el barrio, solo el $24 \%$ de los comercios acepta pagos digitales.

La definición de la Cámara Argentina de FinTech es "Las FinTech son empresas de origen digital cuya actividad principal es brindar servicios financieros mediante el uso de la tecnología", Cámara Argentina de FinTech (2018). El Financial Stability Board define FinTech como "innovación financiera posibilitada por la tecnología que puede resultar en nuevos modelos de negocio, aplicaciones, procesos o productos con un efecto material asociado sobre los mercados e instituciones financieras y la provisión de servicios financieros" (traducción de los autores), http://www.fsb.org/what-we-do/policy-development/additional-policy-areas/monitoring-offintech/.

Una característica que diferencia el modelo de negocios de las firmas digitales y los bancos tradicionales es que el de las primeras está basado en el cliente, en satisfacer sus necesidades y resolver sus problemas, y el de los últimos, en los productos o servicios. 
Nótese que esto es muy diferente a estrategias competitivas por parte de los bancos que innovan e introducen tecnología en la provisión de sus servicios para adaptarse a las nuevas condiciones y no perder clientes. Estas son las reacciones esperables en mercados competitivos y que reguladores y agencias de competencia no deberían entorpecer.

Obsérvese que un factor de éxito fundamental en la implementación de medidas es la colaboración entre la agencia de competencia y el Banco Central. En Greco y Viecens (2019) documentamos la colaboración entre la Comisión Nacional de Defensa de la Competencia (CNDC) y el Banco Central de la República Argentina (BCRA), que fue determinante para el logro de las reformas procompetitivas en Argentina. En Brasil, el Conselho Administrativo de Defesa Econômica (CADE) y el Banco Central do Brasil (BACEN) firmaron un Memorándum de Entendimiento en febrero de 2018 y un Acto Normativo Conjunto en diciembre de 2018 para armonizar y lograr acciones más eficientes en el ámbito en el que ambas instituciones deben actuar (CADE 2019). En COFECE (2014) se destacó también la importancia de la colaboración con reguladores sectoriales, en particular relativa a un flujo de información que permita a la Comisión Federal de Competencia Económica (COFECE) conocer mejor las condiciones de competencia de los mercados e identificación de prácticas anticompetitivas.

El 29 de agosto de 2016 se firmó la Resolución N 17 de la CNDC (disponible en http://cndc. produccion.gob.ar/node/2479), en adelante la Resolución $\mathrm{N}^{\circ} 17$, que incluyó recomendaciones procompetitivas para el Banco Central de la República Argentina (BCRA), y una recomendación para el Secretario de Comercio de abrir una investigación de oficio contra Prisma y todos sus accionistas. La investigación de oficio se abrió el $1^{\text {}}$ de septiembre de 2016. La CNDC recibió un premio del Banco Mundial y la Red Internacional de Agencias por esta investigación y recomendaciones (https://www.argentina.gob.ar/noticias/premian-la-comision-nacional-dedefensa- de-la-competencia-porla-investigacion-sobre-el).

Véase una versión no confidencial del Compromiso presentado por Prisma (en Anexo): http:// cndc.produccion.gob.ar/sites/default/files/cndcfiles/C-1613.pdf. Para detalles de la operación de venta, véanse: Resolución N 781 de 2019 de la Secretaría de Comercio Interior (RESOL-2019- 781-APNSCI\#MPYT) y Dictamen CNDC de fecha 15 de octubre de 2019 (Conc. 1681).

Las instituciones de pago, así como las instituciones financieras, pueden actuar en la emisión de tarjetas de crédito aunque están sujetas a una regulación más liviana (CADE 2019).

Véase: http://www.cade.gov.br/noticias/cade-instaura-processo-contra-quatro-bancos-porsupostadiscriminacao-ao-nubank.

Véase: http://www.cade.gov.br/noticias/tribunal-do-cade-mantem-medida-preventiva-contraitau-e-rede.

En abril de 2020, el TDLC rechazó un acuerdo extrajudicial entre la FNE y Transbank referido al sistema tarifario para el mercado de medios de pago con tarjetas (https://www.tdlc.cl/nuevo_tdlc/tdlc-rechazaacuerdo-extrajudicial-presentado-por-la-fne-y-transbank/). En mayo, el TDLC inició un procedimiento de consulta acerca de la conformidad con la libre competencia del sistema tarifario (https://www.tdlc.cl/nuevo_tdlc/category/lexsoft/noticias/).

Véase la Opinión: https://www.cofece.mx/CFCResoluciones/docs/Opiniones/V20/6/3953499.pdf, por la que COFECE recibió un premio del Banco Mundial y la Red Internacional de Agencias. La distinción

$\uparrow 17$ destacó que las recomendaciones emitidas por COFECE promovieron que una de las primeras leyes en el mundo sobre servicios de tecnología financiera facilite el ingreso efectivo al mercado financiero de nuevos competidores: https://www.cofece.mx/premian-a-cofece-por-laopinion- a-la-ley-fintech/. 
Extracto del acuerdo publicado en el Diario Oficial de la Federación: https://www.cofece. mx/wpcontent/uploads/2018/10/DOF-IEBC-005-2018-26102018.pdf. COFECE destaca: "Se consideran barreras a la competencia cualquier característica estructural del mercado, así como cualquier hecho o acto de agentes económicos que tenga por objeto o efecto impedir el acceso de competidores, que limite su capacidad para competir o que distorsione el proceso de competencia. También las disposiciones jurídicas de cualquier orden de gobierno cuando indebidamente impidan o distorsionen dicho proceso". https://www.cofece.mx/cofece-investigaposibles- barreras-a-la-competencia/.

Véase: https://repositorio.indecopi.gob.pe/handle/11724/6525.

Parte de esta sección ha sido desarrollada por los autores en Greco y Viecens (2019, 2020).

Hay autores que sostienen que el enfoque utilizado por la Corte Suprema de Estados Unidosde definir un único mercado de transacciones no es correcto (Katz y Sallet 2018), y que pueden tenerse en cuenta los efectos indirectos de red entre ambos lados de la plataforma de transacciones definiendo mercados separados (de hecho este criterio es adoptado por el voto en disenso de la Corte Suprema). Para un enfoque coincidente con el fallo de la Corte, véase: Evans y Schmalensee (2019).

Véase Greco y Viecens (2020) para una discusión más desarrollada sobre las diferencias entre el caso AMEX de Estados Unidos y el caso de Argentina.

El TDLC cita una sentencia previa (Sentencia TDLC $N^{\circ}$ 29/2005) en la cual adoptó una definición amplia del mercado bilateral. Sin embargo, el análisis desarrollado en TDLC (2017) da cuenta de la evolución del mercado y de los riesgos para la competencia, y realiza un análisis separado de las distintas actividades, en particular de la falta de competencia en la adquirencia. Este enfoque analítico es coherente con la propuesta de cambio en la estructura de mercado y del modelo de negocios prevaleciente, y con el adoptado en Argentina y Brasil.

Entre VisaNet y Visa existía un contrato; en el caso de Redecard y Mastercard la exclusividad era de hecho.

La posibilidad de los adquirentes de ofrecer diversas marcas de tarjetas de crédito y débito y medios de pago alternativos, de modo que los comercios pudieran tratar con un solo proveedor para los diversos medios de pago y que se desarrollara la competencia entre los adquirentes. El Termo de Compromisso de Cessação (TCC) consiste en un acuerdo celebrado entre CADE y las empresas investigadas por conductas anticompetitivas por el cual la autoridad suspende la investigación y la empresa se compromete al cumplimiento de una serie de obligaciones (CADE 2019).

Véase:

https://www.hacienda.cl/sala-de-prensa/noticias/historico/ministro-de-haciendaanuncia-importantes.html.

Véase: https://www.indecopi.gob.pe/documents/51771/2610439/Sistemas+de+tarjetas+de+pago/.

Se refiere a los protocolos, instrumentos, interfaces, procedimientos, reglas, programas, sistemas, infraestructura y demás elementos que permiten la prestación del servicio de tarjetas, cheques, transferencias y pagos de recursos (COFECE 2014).

Véase nota al pie 28. 
En Argentina, por ejemplo, MercadoLibre se sumó como denunciante a la investigación que la CNDC abrió de oficio, aunque luego retiró la denuncia (Greco y Viecens 2019). Véase también la denuncia de Nubank en CADE (Tabla 2).

Nótese que a escala internacional no existe un único modelo de formación de precios. Hay países (como Estados Unidos) en los que la tasa de intercambio para transacciones con tarjetas de crédito no está regulada. Sin embargo, por las características que mostraba el mercado en Argentina, se consideró que, dados los riesgos de falta de competencia en la determinación de la tasa de intercambio, era preferible "regular para la competencia".

Véase: Comunicación "A" 6212, http://www.bcra.gov.ar/Pdfs/comytexord/A6212.pdf.

Circular № 3.887, de marzo de 2018 del Banco Central do Brasil.

Los cambios en la organización del mercado orientados a la separación de los roles emisor y adquirente han implicado que en la actualidad las tasas de intercambio estén fijadas por las marcas de tarjetas.

El Compromiso abarcó la venta del $100 \%$ de las acciones en dos etapas, la primera implicaba la venta del 51\% y la cesión del control, y la segunda la venta del 49\% restante. Versión no confidencial de aceptación del Compromiso: http://cndc.produccion.gob.ar/sites/default/files/ cndcfiles/C-1613.pdf.

Véase nota al pie 26.

"Si bien, la estructura actual del mercado de medios de pago en Chile ha contribuido a extender la utilización de las tarjetas y sus beneficios, se aleja de los estándares internacionales y representa una barrera de entrada a potenciales nuevos competidores" (Ministro de Hacienda, Felipe Larrain, abril de 2019, https://www.hacienda.cl/sala-de-prensa/noticias/historico/ ministro-de-hacienda-anunciaimportantes.html).

En particular, nótese que Rochet y Tirole (2002) asumen un mercado de adquirencia altamente competitivo y un mercado de emisión con poder de mercado, lo contrario a lo que se observa en mercados como el de Argentina y Chile.

Este dilema o trade-off entre competencia y estabilidad financiera se ha manifestado, por ejemplo, luego de la Gran Depresión de los años 30, cuando se soslayaron los objetivos de competencia e innovación y se permitió una mayor concentración, con una gran participación del Estado en el sistema mediante regulaciones restrictivas (Bofondi y Gobbi 2017).

Se conoce como shadow banking la realización de actividades riesgosas fuera de los registros contables por parte de los bancos -off-balance sheet activities-, mediante una larga cadena de intermediación financiera.

En economía se denomina moral hazard o riesgo moral a una situación en la cual existen asimetrías de información y un agente no asume por completo las consecuencias de sus propias acciones, sino que por el contrario, otros agentes soportan las consecuencias de los riesgos asumidos. 
Véase al respecto: Vives (2019), Bofondi y Gobbi (2017) y las experiencias de Fintech innovation hubs en $\uparrow 43$ Holanda, Italia y USA (Mansilla Fernández 2017) y regulatory sandboxes en UK (UK Financial Conduct Authority 2015).

†4 Véase nota al pie 6. 